\title{
Empreendedorismo e inovação nas cidades inteligentes: uma revisão sistemática da literatura
}

\section{Entrepreneurship and innovation in smart cities: a systematic literature review}

\author{
1 Cleide Mara Barbosa da Cruz cmara.cruz@hotmail.com \\ 2 Cleo Clayton Santos Silva
}

1 Mestra e Doutoranda em Ciência da Propriedade Intelectual pela Universidade Federal de Sergipe, Especialista em Gestão Empresarial e Inteligência Competitiva pela Faculdade Metropolitana do Estado de São Paulo, Bacharela em Administração Pública pela Univer-sidade Federal de Sergipe, Técnica em Secretariado pelo Instituto Federal de Sergipe.

2 Mestrado em Biometria e Estatística Aplicada pela Universidade Federal Rural de Per-nambuco, Bacharel em Estatística pela Universidade Federal de Sergipe, Professor na Faculdade de Administração e Negócios de Sergipe - FANESE.

\section{Resumo}

O crescimento da população urbana é reconhecido como uma tendência que desafia a administração das cidades ao redor do mundo e, nesse contexto, são vários os avanços tecnológicos que estão sendo desenvolvidos para estimular a inovação nos centros urbanos, de forma a tornar as cidades inteligentes, objetivo de todas as cidades modernas. Atualmente já existem muitas cidades que alcançam grande sucesso em alguns campos, principalmente com o uso de tecnologias, visto que os empreendimentos são mais modernos, permitindo que as cidades adiram à inovação tecnológica em diversos âmbitos. No entanto, para as cidades se tornarem inteligentes, é necessário se adequar às mudanças e introduzir inovações e, para isso, é primordial envolver questões empreendedoras para o seu crescimento. 0 objetivo deste estudo foi realizar uma revisão sistemática da literatura sobre o desenvolvimento das cidades inteligentes através do empreendedorismo e inovação, com base em buscas realizadas nas plataformas Science Direct, Scielo e Scopus. A análise dos artigos finalistas da triagem do estudo identificou que o processo de inovação tecnológica e empreendedorismo está diretamente ligado ao sucesso de uma cidade, de forma que seja considerada inteligente. Conclui-se que os principais fatores para o desenvolvimento de uma cidade que almeja se tornar inteligente são: a criatividade agregada à gestão empreendedora, por meio das demandas estruturais, culturais, sociais, econômicas, institucionais e ambientais, bem como o processo de inovação tecnológica e empreendedorismo, pois ambos estão diretamente ligados ao sucesso de uma cidade moderna.

\section{Palavras-chave:}

Cidades inteligentes. Empreendedorismo. Inovação.

\begin{abstract}
The urban population growth is recognized as a trend that challenges the administration of cities around the world and, in this context, there are several technological advances that are being developed to stimulate innovation in urban centers, in order to make cities smart, the goal of all modern cities. Currently, there are already many cities that achieve great success in some fields, mainly with the use of technologies, since the enterprises are more modern, allowing cities to adhere to technological innovation in different areas. However, in order to become smart cities, it is necessary to adapt to changes and introduce innovations, and for that, it is essential to involve entrepreneurs issues for their growth. The aim of this study was to conduct a systematic review of the literature on the development of smart cities through entrepreneurship and innovation, based on searches carried out on Science Direct, Scielo and Scopus platforms. The analysis of the final articles in the study screening identified that the process of technological innovation and entrepreneurship are directly linked to the success of a city, so that it is considered smart. It is concluded that the main factors for the development of a city that aims to become smart are: creativity added to entrepreneurs management, through structural, cultural, social, economic, institutional and environmental demands, as well as the process of technological innovation and entrepreneurship, as both are directly linked to the success of a modern city.
\end{abstract}

\section{Keywords:}

Smart cities. Entrepreneurship. Innovation.

\section{Como você deve citar?}

CRUZ, Cleide Mara Barbosa da; SILVA, Cleo Clayton Santos. Empreendedorismo e inovação nas cidades inteligentes: uma revisão sistemática da literatura. Cadernos UniFOA, Volta Redonda (RJ), v. 16, n. 47, p. 91-100, dez, 2021. 
As cidades ao redor do mundo vivenciam determinadas experiências, pois possuem um percentual elevado de dados gerados por indivíduos, contribuindo de tal forma para gestão, planejamento e participação nas cidades. As dinâmicas urbanas têm sua administração baseada em acesso à informação, pois ocorre o crescimento do planejamento e políticas públicas e, com isso, o interesse da esfera pública tem aumentado nas cidades do mundo. Dessa forma, inicia-se o desenvolvimento das cidades inteligentes (ANTONIALLI; KIRA, 2020).

As cidades contemporâneas possuem um modelo para se tornarem inteligentes, visto que é necessário que abordem novas tecnologias da informação e comunicação, sistemas inteligentes interligados, bem como uma gestão mais eficiente. As cidades estão constantemente se transformando e, consequentemente, atraindo esforços de pesquisadores e oportunidades para implantar novos sistemas, para que se adaptem às possibilidades atuais (OLIVEIRA, 2020).

Com o rápido acúmulo da população e indústria, a eficiência do serviço urbano, os requisitos na construção de cidades sustentáveis e inteligentes estão se tornando cada vez mais rápidos (JIA;WU, 2020).

Atualmente as cidades inteligentes que fazem amplo uso de tecnologias digitais são apontadas como possíveis soluções para as pressões populacionais enfrentadas por muitas cidades. Por isso, é necessário criar parcerias no setor público e privado para realizar a visão de cidade inteligente (TAN; TAEIHAGH, 2020).

O empreendedorismo e inovação estão correlacionados no contexto das cidades e apresentam relação significativa com a dimensão e desempenho econômico, principalmente com a variável da taxa de emprego. Dessa forma, é importante que formuladores de políticas públicas tenham consciência dessa importância e passem a agir no sentido de auxiliar atividades empreendedoras e inovadoras por meio de alternativas (GARCIA, 2019).

Assim, as comunidades urbanas, cada vez mais, estão se dando conta de que precisam buscar novas alternativas para continuar seu desenvolvimento. Nessa perspectiva, a inovação e as tecnologias devem entrar na pauta das cidades, como parte das discussões sobre a gestão urbana, ao mesmo tempo em que existe a concentração urbana que amplia também os problemas. No entanto, o desenvolvimento de novas tecnologias e a complexidade do panorama urbano criaram novas facilidades para transformar a realidade das cidades (KNIESS; PHILIPPI; AGUIAR, 2019).

Considerando esse cenário, este artigo tem como objetivo realizar uma revisão sistemática da literatura (RSL) sobre o desenvolvimento das cidades inteligentes por meio do empreendedorismo e da inovação. Essa revisão RSL se deu através de buscas realizadas nas plataformas Science Direct, Scielo e Scopus e, por meio dos artigos finalistas, foi possível verificar se existe alguma correlação entre o processo de inovação tecnológica e empreendedorismo para que uma cidade possa receber a nomenclatura de cidade inteligente.

\section{REVISÃO DA LITERATURA}

Esta seção está dividida em três subseções, a saber: cidades inteligentes; empreendedorismo e inovação nas cidades; desafios e perspectivas para aplicação do empreendedorismo e inovação nas cidades inteligentes. 


\subsection{Cidades Inteligentes}

O surgimento das cidades inteligentes ocorre por meio da eficiência nas dinâmicas urbanas, ou seja, através do uso das novas tecnologias digitais. 0 urbanismo do século XXI aborda a cidade inteligente como uma área marcada e governada por meios tecnológicos capazes de possibilitar fácil acesso a informações, bem como eficiência na gestão (SIMÃO; FIRMINO, 2019). Por isso, para se entender de forma clara o conceito das cidades inteligentes, é necessário, não apenas analisar a Ciência da Informação, mas considerar conhecimentos multidisciplinares (FERNANDES, 2019).

O discurso da cidade inteligente pode dizer muito sobre o urbanismo contemporâneo. Esse discurso é indiscutivelmente um exemplo-chave da característica cada vez mais móvel e em rede de reformulação de políticas urbanas, revelando insights importantes sobre os processos políticos que atualmente moldam as cidades (WATHNE; HAARSTAD, 2020). 0 conceito de cidade inteligente é um dos mais importante e altamente desenvolvidos, para melhorar a qualidade de vida e a eficiência de determinada população, já que os sistemas apresentam uma comunicação entre empresas e clientes. (NASCIMENTO; SOUZA; SERRALVO, 2018).

\subsection{Empreendedorismo e Inovação nas cidades}

O empreendedorismo ocorre para que exista a interdependência, de forma que as localidades inseridas sejam capazes de disseminar suas potencialidades e gerar empresas de caráter tecnológico (FIGUEIREDO; LEITE, 2006). Com a evolução dos estudos, os empreendedores variam entre si de forma significativa e essas variações apresentam ampla gama de implicações sobre o comportamento e desempenho dos empreendimentos, em termos de papéis desempenhados, atributos pessoais, modelos de gestão e capitais econômicos, sociais e simbólicos mobilizados que convivem em constante inter-relação, conflito e aliança no seio dos processos de transformações vivenciadas pelas cidades (SANT'ANNA; NELSON, 2013).

A orientação empreendedora retrata elementos distintos, como inovabilidade, sendo complementar para o empreendedorismo nas organizações, considerando o conceito de inovar, postura de se engajar e apoiar novas ideias, novos experimentos e processos criativos que resultem em novos produtos, serviços ou processos (MARTENS; FREITAS; ANDRES, 2011).

Os empreendedores são os agentes responsáveis pela inovação, associados às forças direcionadas de forma a promover o desenvolvimento econômico a partir de ideias e práticas. Nesse contexto, a função empreendedora não está incorporada somente em uma pessoa física, pois considera o ambiente, o contexto e os diferentes setores econômicos nos quais podem vigorar ações que abram alternativas de observação do fenômeno empreendedorismo (GOMES; LIMA; CAPPELLE, 2013).

O empreendedorismo tem o objetivo de ajudar a determinar o ponto de partida das cidades, bem como o processo de transformação planejada em um nível consciente, tornando, dessa forma, a cidade mais inteligente (GOTLIB, 2020). Para criar uma cidade verdadeiramente inteligente, é necessário entender a cidade como um complexo, e, para isso, são necessárias leis, termos, tecnologias de produção inteligentes, internet segura, conexão e auditorias, para que as pessoas desenvolvam seus empreendimentos e invistam sem receio, apoiando investimentos e crescimento econômico da cidade (RISTVEJ; LACINÁK; ONDREJKA, 2020).

As novidades divulgadas acabam gerando necessidades à população, pois estimulam os indivíduos para o consumo, ou ainda, a descobrirem vantagens no aperfeiçoamento. Nesse sentido, as dinâmicas 
econômicas são a inovação e a qualidade (ENDLICH, 2007), considerando o conceito de inovação já abordado (MARTENS; FREITAS; ANDRES, 2011).

O crescimento econômico e as reformas urbanas contribuem para solucionar problemas socioeconômicos das cidades e incentivam iniciativas empreendedoras para o fomento do desenvolvimento com empresas de caráter tecnológico. Por isso, as reformas urbanas disseminam o crescimento das cidades, acarretando em surgimento de empresas de caráter tecnológico com maior vantagem competitiva, se comparada a outras empresas (FIGUEIREDO; LEITE, 2006).

As cidades têm papel fundamental como ambiente rico e propício ao desenvolvimento de polos de inovação, tornando-se catalisadoras de requalificação urbana. Algumas cidades elaboram estratégias para serem catalisadoras de inovações tecnológicas na sociedade da informação, articulando atores públicos e privados (DUARTE, 2005). Assim, a importância de as cidades inteligentes participarem de redes de transferência de conhecimento e tecnologias tem o intuito de otimizar os seus processos e resultados, para que, dessa forma, possibilitem a resolução de problemas de diferentes complexidades por meio da inovação (KNIESS; PHILIPPI; AGUIAR, 2019).

\subsection{Desafios e perspectivas para aplicação do empreendedorismo e inovação nas cidades inteligentes}

O termo "cidade inteligente" surgiu como uma nova dimensão de gestão pública, para o enfrentamento dos desafios existenciais (WEISS; BERNARDES; CONSONI, 2015). As necessidades emergentes dos cidadãos nas sociedades modernas e a crescente migração para grandes centros urbanos são desafios cruciais para os gestores públicos, visto que o governo tem o potencial de basear suas estratégias para a prestação de serviços públicos em alternativas colaborativas, promovendo a coprodução como uma solução gerencial inteligente para as cidades, iniciada por poucos segmentos, no sentido de estabelecer cidades inteligentes (COUTINHO, 2019).

Para que as cidades não enfrentem tantos desafios na transformação, é necessário que exista comunicação, interação, envolvimento e contribuições, vitais para se alcançar objetivos das cidades inteligentes. Essas iniciativas são essenciais para garantir um futuro melhor com rápida expansão global e, apesar dos desafios significativos a serem enfrentados, podem também apresentar um impacto real (HASIJA; SHEN, TEO, 2020).

Nesse contexto, existe a necessidade de se estimular os negócios das cidades e fomentar áreas empreendedoras como uma fonte de renovação e, para isso, é necessário que os atores locais priorizem determinadas ações. Por isso, é indispensável identificar a inovação e ter a mentalidade empreendedora, de forma a influenciar as empresas e empreendedores para o desenvolvimento das cidades (NATÁRIO; BRAGA; FERNANDES, 2018).

As cidades inteligentes possuem correlação com programas de inovação urbana, bem como os ambientes físicos e virtuais para lidar com novas tecnologias e, ao mesmo tempo, empreender. Do ponto de vista das inovações urbanas, visando ao desenvolvimento, seria importante dar mais atenção na avaliação e impacto da gestão das cidades, pois essa inovação possui alguns desafios que devem ser enfrentados, tais como redução das desigualdades sociais no acesso à tecnologia, transferência de tecnologia para as nações com menos recursos financeiros e adoção de novos modelos de financiamento nas cidades (KNIESS; PHILIPPI; AGUIAR, 2019). 
Trata-se de uma revisão sistemática da literatura. De acordo com Pereira e Galvão (2014), revisões sistemáticas são classificadas como estudos secundários que possuem sua fonte de dados nos estudos primários, ou seja, os estudos primários são constituídos por artigos científicos que mostram os resultados da pesquisa em primeira mão.

Para a pesquisa em questão, buscou-se, nas fontes de pesquisa, a seleção das publicações de acordo com os critérios de inclusão e exclusão, realizada nas plataformas de buscas Science Direct, Scielo e Scopus, utilizando-se como palavras-chave os termos "empreendedorismo", "inovação" e "cidades inteligentes", em Língua Portuguesa, como disposto no Quadro 1, na seção de análise dos resultados. A justificativa da escolha das bases citadas se deu com o intuito de se verificar a relação do tema com o conteúdo, através das palavras-chave mencionadas.

Figura 1 - Processo de protocolo organizado para o estudo

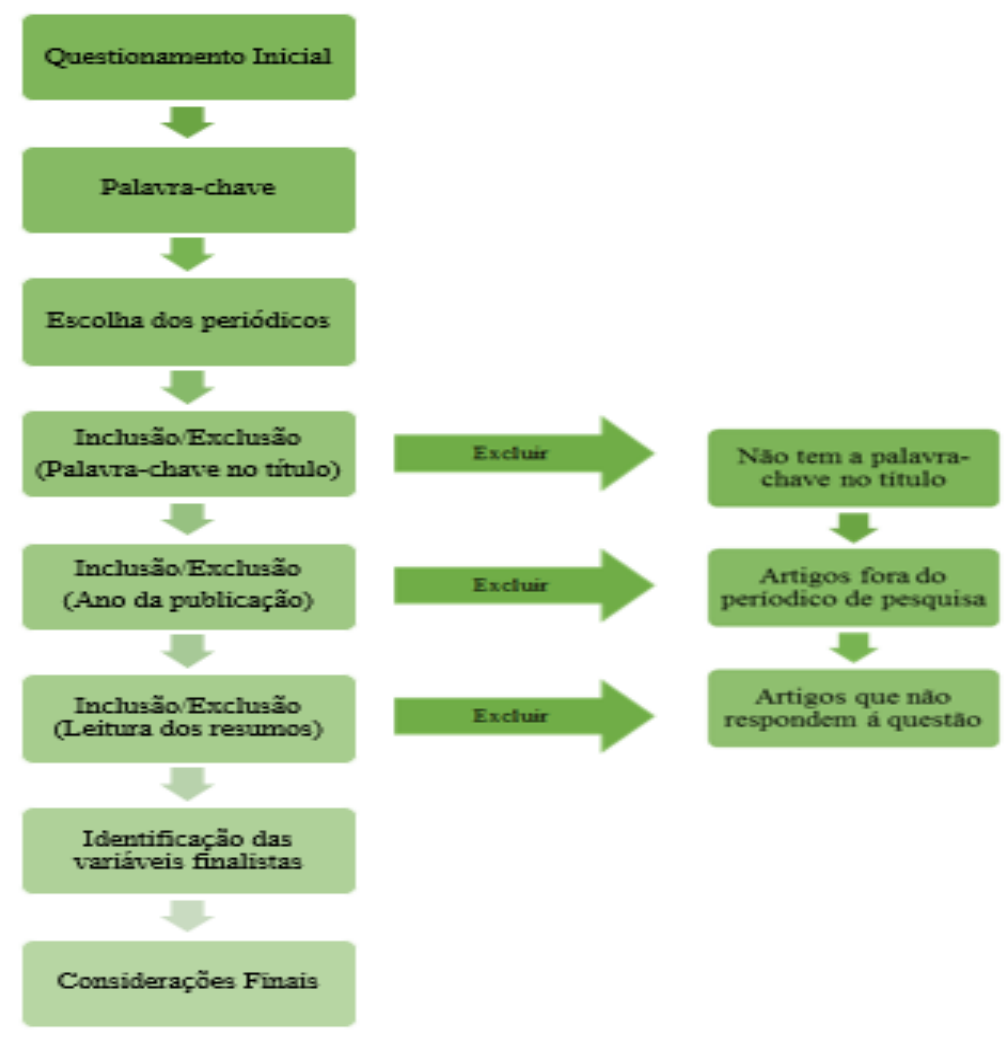

Fonte: Elaborado pelos autores (2020)

A Figura 1 explicita o protocolo de inclusão e exclusão organizado para o estudo, pontuando alguns critérios descritos neste trabalho. Os critérios de inclusão adotados foram: a) revisões de literatura ou sistemáticas sobre cidades inteligentes; $b$ ) textos publicados em português; $c$ ) publicações realizadas no período compreendido entre 2009 e 2019; d) título e/ou resumo contendo as palavras-chave de pesquisa. Os critérios de exclusão adotados foram: a) artigos repetidos; b) pesquisas anteriores ao ano de 2009 e pesquisas aceitas e publicadas em 2020; c) título e resumo sem palavras selecionadas ou diferentes do contexto a ser pesquisado. 


\section{RESULTADOS E DISCUSSÃO}

A busca inicial identificou cinco mil seiscentos e dezoito (5.618) artigos nas bases de dados, sendo: na Science Direct, mil setecentos e quarenta e oito (1.748); na Scielo, três mil setecentos e sessenta e seis (3.766); e na Scopus, cento e quatro (104), no espaço temporal compreendido de 2009 a 2019. $\mathrm{Na}$ análise dos resumos, somente oito (08) artigos foram incluídos para avaliação do texto completo. A seleção final foi baseada na relevância, elegibilidade e desenho do estudo, verificando-se quais artigos atendiam à pergunta norteadora do estudo. Dessa forma, apenas quatro (04) artigos se encaixavam nas questões mencionadas.

Quadro 1: Busca inicial com base nas palavras-chave em Língua Portuguesa

\begin{tabular}{|c|c|c|c|}
\hline Bases & Empreendedorismo & Inovação & Cidades Inteligentes \\
\hline Science Direct & 122 & 1603 & 23 \\
\hline Scielo & 464 & 3275 & 27 \\
\hline Scopus & 73 & 26 & 05 \\
\hline Total & 659 & 4904 & 55 \\
\hline
\end{tabular}

Fonte: Elaborado pelos autores (2021)

O Quadro 1 mostra o quantitativo de artigos com base nas palavras-chave que foram encontradas em Língua Portuguesa e os seus respectivos filtros.

Quadro 2: Fase das palavras-chave no título e texto do tipo artigo em Língua Portuguesa

\begin{tabular}{|c|c|c|c|}
\hline Bases & Empreendedorismo & Inovação & Cidades Inteligentes \\
\hline Science Direct & 08 & 119 & 00 \\
\hline Scielo & 81 & 297 & 06 \\
\hline Scopus & 58 & 08 & 00 \\
\hline Total & 147 & 424 & 06 \\
\hline
\end{tabular}

Fonte: Elaborado pelos autores (2021)

O Quadro 2 evidencia os artigos identificados e escolhidos com base na palavra-chave no título/ resumo do artigo. Com o uso dos filtros, essa busca resultou em quinhentos e setenta e sete (577) artigos científicos.

Quadro 3: Análise dos resumos

\begin{tabular}{|c|c|c|c|}
\hline Bases & Empreendedorismo & Inovação & Cidades Inteligentes \\
\hline Science Direct & 00 & 04 & 00 \\
\hline Scielo & 00 & 02 & 02 \\
\hline Scopus & 00 & 00 & 00 \\
\hline Total & 00 & 06 & 02 \\
\hline
\end{tabular}

Fonte: Elaborado pelos autores (2021)

O Quadro 3 apresenta os artigos finalistas para o estudo. Dos vários fatores que contribuíram para que os oito (08) se destacassem, a questão de inovação no setor empreendedorismo chamou atenção na análise dos resumos, para o fato de verificar se os artigos correspondiam às cidades inteligentes. Dessa forma, com relação ao texto completo, optou-se por excluir quatro (04) artigos, pelo fato de que, 
na leitura, o fator ligado ao tema não estava correlacionado com as cidades inteligentes, ou seja, a inovação e o empreendedorismo estavam presentes no texto, mas, no quesito aplicação em cidades, não houve correlação. Logo, não respondiam à questão norteadora do referido estudo.

O Quadro 4, resultante das variáveis dos artigos correspondentes à questão norteadora da busca, inicialmente tratada em seus critérios de inclusão e exclusão, mostra que quatro (04) artigos atendiam à pergunta do estudo, cujo objetivo principal, além da realização da revisão sistemática de literatura, era verificar a aplicação do empreendedorismo e inovação para o desenvolvimento de cidades inteligentes.

Em um dos artigos selecionados, os autores, Piekas et al. (2018) confirmam, em seus resultados, que uma cidade inteligente e criativa deve possuir determinadas diretrizes e considerar suas demandas culturais, sociais, econômicas, institucionais e ambientais. Dessa forma, a transição é um processo em curso desde que essas demandas sejam atendidas.

Quadro 4 - Variáveis dos artigos correspondentes à questão norteadora

\begin{tabular}{|c|c|c|c|c|c|}
\hline Título & Autores & Palavras- chave & Revista & Ano & Método \\
\hline $\begin{array}{l}\text { Aspectos legais e } \\
\text { percepções sobre } \\
\text { as estratégias para } \\
\text { cidades inteligentes } \\
\text { e criativas: estudo } \\
\text { da cidade de } \\
\text { Chapecó (SC) }\end{array}$ & $\begin{array}{l}\text { Andrezza } \\
\text { Aparecida Saraiva } \\
\text { Piekas, Rógis } \\
\text { Juarez Bernardy, } \\
\text { Simone Sehnem, } \\
\text { Juliana Fabris }\end{array}$ & $\begin{array}{l}\text { Cidades inteligentes. } \\
\text { Cidades criativas. } \\
\text { Desenvolvimento }\end{array}$ & $\begin{array}{l}\text { Revista Brasileira } \\
\text { de Gestão Urbana } \\
\text { (Brazilian Journal of } \\
\text { Urban Management) }\end{array}$ & 2018 & $\begin{array}{l}\text { Estudo de caso } \\
\text { qualitativo }\end{array}$ \\
\hline $\begin{array}{l}\text { Cidades inteligentes } \\
\text { como nova } \\
\text { prática para o } \\
\text { gerenciamento } \\
\text { dos serviços e } \\
\text { infraestruturas } \\
\text { urbanos: a } \\
\text { experiência da } \\
\text { cidade de Porto } \\
\text { Alegre }\end{array}$ & $\begin{array}{l}\text { Marcos Cesar } \\
\text { Weiss, Roberto } \\
\text { Carlos Bernardes, } \\
\text { Flavia Luciane } \\
\text { Consoni }\end{array}$ & $\begin{array}{l}\text { Cidades inteligentes. } \\
\text { Cidades digitais. } \\
\text { Gestão da inovação } \\
\text { tecnológica das } \\
\text { cidades. }\end{array}$ & $\begin{array}{l}\text { Revista Brasileira } \\
\text { de Gestão Urbana } \\
\text { (Brazilian Journal of } \\
\text { Urban Management) }\end{array}$ & 2015 & $\begin{array}{l}\text { Qualitativo e } \\
\text { exploratório, } \\
\text { baseado em } \\
\text { estudo de caso } \\
\text { com coleta de } \\
\text { dados }\end{array}$ \\
\hline $\begin{array}{l}\text { Política Urbana } \\
\text { de Inovação } \\
\text { Tecnológica: } \\
\text { Experiências De } \\
\text { Cidades Digitais No } \\
\text { Brasil }\end{array}$ & $\begin{array}{l}\text { Aldenilson Costa, } \\
\text { Tamara Egler, } \\
\text { Antònia Casellas }\end{array}$ & $\begin{array}{l}\text { Política pública; } \\
\text { inovação } \\
\text { tecnológica; cidade } \\
\text { digital; digitalização }\end{array}$ & Finisterra & 2019 & $\begin{array}{l}\text { Levantamento } \\
\text { bibliográfico }\end{array}$ \\
\hline $\begin{array}{l}\text { Empreendedores } \\
\text { cívicos e Smart } \\
\text { Cities: práticas, } \\
\text { motivações e } \\
\text { geografias da } \\
\text { inovação }\end{array}$ & $\begin{array}{l}\text { Luiz Carvalho e } \\
\text { Catarina Maia }\end{array}$ & $\begin{array}{l}\text { Smart City; } \\
\text { Empreendedores } \\
\text { Cívicos; Dados } \\
\text { Abertos; Geografia } \\
\text { Económica }\end{array}$ & $\begin{array}{l}\text { Revista de Geografia } \\
\text { e Ordenamento do } \\
\text { Território (GOT) }\end{array}$ & 2016 & $\begin{array}{l}\text { Revisão de } \\
\text { literatura }\end{array}$ \\
\hline
\end{tabular}

Fonte: Elaborado pelos autores (2021)

Conforme os autores Weiss, Bernardes e Consoni (2015), por meio da sua pesquisa qualitativa baseada em um estudo de caso, a experiência da cidade de Porto Alegre, com referência à materialização do conceito de cidade inteligente, mostrou que as tecnologias da informação e comunicação inseridas nas cidades acarretam na eficiência da gestão, ressaltando-se que a implementação das TICs não é solução única para resolução dos problemas nesses serviços. 
De acordo com Costa, Egler e Casellas (2019), em seu levantamento bibliográfico sobre inovação tecnológica e experiências de cidades digitais no Brasil, foi possível demonstrar que as bases para uma cidade ser reconhecida como inteligente se baseia na modernização da burocracia do estado com relação à promoção da inclusão digital e ao fornecimento de serviços públicos por meio de tecnologias digitais. Dessa forma, as experiências das cidades de São Paulo, Sud Menucci, Porto Alegre, Palmas e Piraí expuseram aspectos com relação aos arranjos institucionais em virtude da implantação de políticas públicas, bem como os empecilhos orçamentários que determinadas cidades possuem.

Carvalho e Maia (2016) buscam uma reflexão sobre a relevância potencial do território e da geografia nos seus processos de criação de conhecimento e inovação, e, por meio dela, mostram que os empreendedores cívicos têm grande potencial na contribuição para a construção de cidades inteligentes com mais possibilidades de inclusão, inovação, democratização e adaptação para novos desafios das cidades do futuro, criando expectativas positivas no aumento da economia e da inovação de várias cidades.

Com as referências bibliográficas encontradas nas fontes de buscas citadas na metodologia deste estudo, foi possível realizar a proposta embasada na discussão sobre as cidades inteligentes e empreendedoras por meio dos processos de inovação tecnológica presentes em cada artigo finalista analisado.

\section{CONSIDERAÇÕES FINAIS}

O Brasil pode realizar a construção de uma sociedade empreendedora que fomentará o crescimento nacional, sedimentado sobre a inovação e desenvolvimento das empresas de base tecnológica.

Neste estudo, o uso da técnica de revisão sistemática de literatura permitiu a realização de uma síntese do que está sendo desenvolvido no quesito inovação e empreendedorismo nas cidades inteligentes, o que resultou em um número pequeno de artigos que responderam à questão norteadora, porém esses resultados forneceram informações relevantes.

Dessa forma, os resultados encontrados mostraram que os principais fatores para que uma cidade seja considerada inteligente estão ligados à criatividade agregada à gestão empreendedora, por meio das demandas estruturais, culturais, sociais, econômicas, institucionais e ambientais, considerando também a inovação nas tecnologias da informação e comunicação que proporcionam maior eficiência na gestão empreendedora da cidade.

Foi possível a identificação de iniciativas de promoção da inclusão digital e do fornecimento de serviços públicos por meio de tecnologias digitais na contribuição para o desenvolvimento de cidades inteligentes com mais possibilidades de inclusão, inovação, democratização e adaptação para novos desafios das cidades do futuro.

Portanto, o processo de inovação tecnológica e o empreendedorismo estão diretamente ligados ao sucesso de uma cidade, para que seja possível receber a nomenclatura de cidade inteligente, ou seja, uma cidade pensada e preparada com estratégias empreendedoras que utilizem diversas tecnologias inovadoras poderá ser considerada uma cidade do futuro. 


\section{REFERÊNCIAS}

ANTONIALLI, D. M.; KIRA, B. Planejamento Urbano do Futuro, dados do presente: a proteção da Privacidade no Contexto das Cidades Inteligentes. Revista Brasileira de Estudos Urbanos e Regionais, v.22, pg. 1-25, 2020.

CARVALHO, L. MAIA, C. Empreendedores cívicos e Smart Cities: práticas, motivações e geografias da inovação. Revista de Geografia e Ordenamento do Território (GOT), n. 10, 2016COSTA, A.; EGLER, T. CASELLAS, A. Política Urbana De Inovação Tecnológica: Experiências De Cidades Digitais No Brasil. Finisterra, v. 110, pg. 93-113, 2019.

COUTINHO, M. M.Coproduction Between Government and Civil Society to Establish Smart Cities in the State of Pará. RAC, Maringá, v. 23, n. 5, SI Technology Amazon, art. 3, pg. 636-653, setembro/outubro, 2019.

DUARTE, F. Cidades inteligentes: inovação tecnológica no meio urbano. São Paulo: Perspec. [online], vol.19, n.1, pg.122-131, 2005.

ENDLICH, A. M. Novos referenciais de desenvolvimento e planejamento territorial: possibilidades para as pequenas cidades? Redes, Santa Cruz do Sul, v. 12, n. 2, p. 5-35, mai./ago. 2007.

FERNANDES, R.A.S. Urban governance in Latin America: Bibliometrics applied to the context of smart cities. Transinformação, v.31, 2019.

FIGUEIREDO, M. D.; LEITE, E. F. Cidades Empreendedoras: as novas visões sobre planejamento urbano e desenvolvimento econômico no brasil. READ: Revista Eletrônica de Administração, vol. 12, n. 5, p. 268291, set./out. 2006.

GARCIA, Beatriz. Internacionalização, inovação, empreendedorismo e sua relação com o desempenho econômico de cidades: um estudo com cidades do Estado de São Paulo. Dissertação (mestrado) Universidade Estadual de Campinas, Faculdade de Ciências Aplicadas, Limeira, SP, 2019.

GOMES, A. F., De LIMA, J. B.; CAPELLE, M. C. A. Do Empreendedorismo a Noção de Ações Empreendedoras: Reflexões Teóricas. Revista Alcance, vol. 20, núm. 2, abril-junho, 2013.

GOTLIB, D. et al. Multiagency Modeling of Transformation Strategies Towards Sustainable Smart Cities. Appl. Sci. v. 10, 853, 2020.

HASIJA, S., SHEN, Z. J. M., TEO, C. P. Smart City Operations: Modeling Challenges and Opportunities. Manufacturing \& Service Operations Management, v.22, 2020.

JIA, J., WU, X. A Multidimensional Assessment Model Using RE-3DSG Sensors on Net ES and GVR for Sustainable and Smart Cities. Sensors, 20, 1259, pp. 1-16, 2020.

NASCIMENTO, B. N., SOUZA, C. L., SERRAVALVO, F. A. A systematic review of smart cities and the internet of things as a research topic. Cad. EBAPE.BR, v. 17, $n^{\circ} 4,2019$.

KNIESS, C., PHILLIPI Jr., A., AGUIAR, A., \& Conti, D. Inovação urbana e recursos humanos para gestão de cidades sustentáveis. Estudos Avançados, v. 33, 2019. 
MARTENS, C.D.P.; FREITAS, H.M.R.; ANDRES, R. Desenvolvimento da orientação empreendedora em empresas de software: proposições preliminares. Revista Eletrônica de Administração (REAd), v.7, n.2, p. 424-450, 2011.

NATÁRIO, M. M., BRAGA, A. M., \& FERNANDES, G. P. Determinantes do desempenho empreendedor e inovador nas regiões transfronteiriças. Innovar, 28(70), 39-54, 2018.

OLIVEIRA, T. A. Mobility, Citizens, Innovation and Technology in Digital and Smart Cities. Future Internet, v. $12,2020$.

PEREIRA, M. G., \& GALVÃO, T. F. Etapas de busca e seleção de artigos em revisões sistemáticas da literatura. Epidemiologia e Serviços de Saúde, 23, 369-371, 2014.

PIEKAS, A. A. S., BERNARDI, R. J., SEHNEM, S., \& FABRIS, J. Aspectos legais e percepções sobre as estratégias para cidades inteligentes e criativas: estudo da cidade de Chapecó (SC). Revista Brasileira de Gestão Urbana (Brazilian Journal of Urban Management), v. 10, 2018.

RISTVEJ, J., LACINÁK, M. \& ONDREJKA, R. On Smart City and Safe City Concepts. Mobile Netw Appl, 2020.

SANT'ANNA, A. S.; NELSON, R. E. Reconversão de funções econômicas de cidades: contribuições aos estudos sobre empreendedorismo. Pretexto, v. 14, p. 81-97, 2013.

SIMÃO, M. M. B., FIRMINO, R. J. A construção social de um sistema de mobilidade inteligente: mapeando controvérsias no caso do Swisspass. Caderno Metropolitano São Paulo, v. 21, n. 44, pp. 331-354, 2019.

TAN, S. Y., TAEIGHHD, A. Smart City Governance in Developing Countries: A Systematic Literature Review. Sustainability, v. 12, 899, p. 1-29, 2020.

WATHNE, M.W., HAARSTAD, H. The smart city as mobile policy: Insights on contemporary urbanism. Geoforum, v. 108, 2020.

WEISS, M. C., BERNARDES, R. C., \& CONSONI, F. L. Cidades inteligentes como nova prática para o gerenciamento dos serviços e infraestruturas urbanos. Revista Brasileira de Gestão Urbana (Brazilian Journal of Urban Management), v. 7, p. 310-324, 2015. 